\title{
Shedding light on the psychological and behavioral determinants of travel mode choice: A meta-analysis
}

\author{
Pietro Lanzini*, Sana Akbar Khan \\ Department of Management, Università Ca' Foscari, Cannaregio 873, 30121 Venezia, Italy
}

\section{A R T I C L E I N F O}

\section{Article history:}

Received 9 August 2016

Received in revised form 15 November 2016

Accepted 24 April 2017

\section{Keywords:}

Travel mode

Mobility

Meta-analysis

\begin{abstract}
A B S T R A C T
Mobility represents a relevant topic from the standpoint of environmental degradation, health-related consequences and social inclusion. Since private mobility is responsible for the greatest share of polluting emissions, it is necessary to gain deeper understanding of the mechanisms underpinning the choice of individuals to use either cars or alternative, environment-friendly transport modes. A meta-analysis on 58 primary studies is conducted to synthesize evidence on the determinants of travel mode choice, as regards both behavioral intentions and actual behaviors. Results suggest that, besides intentions, habits and past use represent the most relevant predictor, followed by constructs referring to the Theory of Planned Behavior framework. Environmental variables, on the other hand, play a relevant role in shaping behavioral intentions while their effect on actual behaviors is negligible, so that a deep intention behavior gap emerges. A moderator analysis is performed to explain the high heterogeneity in the results. Behaviors' operationalization and measurement emerges as the moderator affecting heterogeneity of outcomes the most; trip purpose, sample type and year of the study also show a moderate effect on heterogeneity, while location does not appear to be a relevant moderator.
\end{abstract}

(c) 2017 Elsevier Ltd. All rights reserved.

\section{Introduction}

There is wide consensus over the un-sustainability of current mobility patterns, and the need to shift towards new paradigms (Collins \& Chambers, 2005; Gardner \& Stern, 2008; Stern, 2011). The transport sector is indeed responsible for problems ranging from air pollution and climate change (Oskamp, 2000) to health related issues (Peters et al., 2004), and even to social exclusion/accessibility (Geurs \& Van Wee, 2004).

Transportation currently accounts for around 14\% of greenhouse gas (GHG) emissions on global scale (IPCC, 2014). In the EU 28, transport in 2013 accounted for 22.2\% of GHG emissions, up from 14.9\% in 1990 (Eurostat tables ${ }^{1}$ ). Moreover, unlike other industrial sectors, transport did not reduce emissions although after the peak of 2007 the trend started to change due to increasing oil prices and diminishing activity by freight vehicles as a consequence of the economic downturn. Similarly in the US, transport accounted for 26\% of GHG emissions in 2014, with a sensible increase since 1990 (EPA, 2016). Until recently, the environmental impacts of transportation have been an issue affecting western countries. However, emerging economies are experiencing a steady increase so that the contribution to emissions deriving from transport sector is bound to rise over the

\footnotetext{
* Corresponding author.

E-mail address: lanzini@unive.it (P. Lanzini).

${ }^{1}$ Eurostat, European Environment Agency, European Topic Centre on Air and Climate Change http://ec.europa.eu/eurostat/statistics-explained/index.php/ Greenhouse_gas_emission_statistics.
} 
next years. China represents a striking example (Gambhir, Lawrence, Tong, \& Martinez-Botas, 2015): vehicle sales rose from 2.1 million in 2000 to 23.5 million in 2014 (CAAM, 2015), with private vehicles and freight respectively responsible for 5\% and $8 \%$ of GHG emissions, and on the increase (Hao, Geng, Li, \& Guo, 2015; Hao, Liu, Zhao, Li, \& Hang, 2015). India has still low figures as regards private cars (with on the other hand many two-wheeler vehicles), yet it is projected to become the third world's largest automobile market, with a rapid growth especially in the segment of small vehicles (Altenburg, Schamp, \& Chaudhary, 2015).

The shift towards sustainable mobility represents a complex issue where various solutions and pathways (either in synergy or in alternative) can be envisaged, encompassing an active role played by different actors and stakeholders. For instance, the automotive industry can propose new or improved technologies capable of curbing the environmental impacts of mobility (e.g., new vehicles such as EVs or the improvement of the efficiency of conventional engines). Local authorities can adopt plans for sustainable mobility in urban areas (like so called SUMPs, Sustainable Urban Mobility Plans) focusing on new infrastructures, improved public transportation or even congestion charges. Policy makers at national and international level can implement standards and regulations to drive the change by means of a top-down approach (e.g., the Fuel Quality Directive, European Commission, 2009). However, citizens represent the key-actor whose involvement is necessary for any sustainable mobility strategy to succeed (Donald, Cooper, \& Conchie, 2014): private mobility is a crucial contributor of $\mathrm{CO}_{2}$ and other pollutants' emissions with detrimental impacts especially in urban areas (Dulal \& Akbar, 2013), and psychological drivers of behavioral change proved to be more effective than infrastructural changes in addressing the issue (Hunecke, Haustein, Böhler, \& Grischkat, 2010). Indeed, there is growing awareness that transport policies aiming at reducing car use can be accomplished by focusing on the psychological constructs of commuters (Möser \& Bamberg, 2008). It is hence necessary to understand the relevance of different drivers capable of spurring the adoption of sustainable mobility patterns.

The present study focuses on the determinants of travel mode choice and the psychological and behavioral correlates of car vs. non-car use. Since existing literature is not conclusive and different studies reach inconsistent results on the main predictors of (sustainable) mobility, we perform a meta-analysis to synthesize existing quantitative research on the topic. To the knowledge of the authors, only one comprehensive meta-analysis on travel modes has been carried out, based on a 2006 database (Gardner \& Abraham, 2008) and representing the starting-point of the present research. Indeed, our study provides a contribution to the ongoing debate by (i) including recent and current research, (ii) broadening the scope of analysis as to encompass further predictors and new perspectives of analysis (which will be described in the methods section) and (iii) investigating possible explanations of the variability across studies, by means of heterogeneity analysis. Our concluding remarks highlight the implications of the results of the meta-analysis, and propose preliminary ideas for future research.

\section{Theoretical models}

Different theoretical frameworks have been applied to investigate travel mode choice, with different degrees of complexity and predictive capability, the most popular of which is represented by the Theory of Planned Behavior (TPB, Ajzen, 1991). TPB is broad in scope and is not born out of environmental research; however, it is very useful to investigate sustainability related domains, including mobility. The theory holds that intentions are the closest antecedents of behavior and have, in turn, three main predictors: attitudes, subjective norms and perceived behavioral control (PBC). Attitudes represent the personal desirability of a behavior, or the feeling of being more or less favorable towards performing the activity. As regards mobility, I might have a positive attitude towards, say, commuting by means of public transportation because I believe that it is nice to contribute to environmental protection through my daily activities. Subjective norms refer to the social pressure we experience: do people who are relevant to me expect that I adopt a specific behavior? That is, for instance: do I feel pressure from my peers and relevant ones to commute by means of environment-friendly transport modes? PBC has been added to the original framework of the Theory of Reasoned Action (TRA, Fishbein \& Ajzen, 1975) as a third predictor of behavioral intentions (and thus behaviors): it accounts for the perceptions of how difficult or easy it is to perform a behavior, representing the answer to speculations that behaviors are not completely under volitional control as originally suggested by TRA. In our example, I might hold positive attitudes and feel social pressure towards sustainable means of commuting, yet I might feel that such behavior is too difficult to adopt, this leading to an attitude-behavior gap (Kollmuss \& Agyeman, 2002; Lane \& Potter, 2007).

TPB has been adopted by a number of studies analyzing the determinants of travel mode (Harland, Staats, \& Wilke, 1999; Klöckner \& Matthies, 2009; Lois, Moriano, \& Rondinella, 2015; Noblet, Thøgersen, \& Teisl, 2014; Nordfjærn, Şimşekoğlu, \& Rundmo, 2014; Polk, 2003). Further variables have been included to integrate the original framework, as to increase the explanatory power of the model: for instance, we can here mention habits (Bamberg \& Schmidt, 2003; Donald et al., 2014; Verplanken, Aarts, van Knippenberg, \& Moonen, 1998), role beliefs (Bamberg \& Schmidt, 2003), personal norms (Manstead \& Parker, 1995; Parker, Manstead, \& Stradling, 1995) and descriptive norms (Donald et al., 2014; Heath \& Gifford, 2002). While the predictive capability of TPB proved to be good (Armitage \& Conner, 2001; Sutton, 1998), the relative importance of the constructs as antecedents of travel mode choice varies across studies (Gardner \& Abraham, 2008).

A second stream of research on transport mode focuses on "feelings of moral obligation to perform or refrain from specific actions" (Schwartz \& Howard, 1981, page 191). Such constructs, which have been suggested as a relevant driver of proenvironmental behaviors, have been labeled as personal norms, moral norms or other equivalent formulations (Conner \& 
Armitage, 1998). According to Norm-Activation-Theory or Model (NAM, Schwartz, 1977), personal norms get activated by variables such as awareness of the adverse consequences of not adopting the virtuous behavior (awareness of consequences) or the ascription of responsibility reflecting feelings of being accountable for such negative outcome (ascription of responsibility).

Moral obligations represent the basis of other psychological theories on consumer behavior such as Value-Belief-Norm (VBN) Theory (Stern, 2000; Stern, Dietz, Abel, Guagnano, \& Kalof, 1999), which integrates the work of Schwartz on values (Schwartz, 1992), NAM and the New Ecological Paradigm (NEP, Dunlap \& Van Liere, 1978). In the words of Schwartz (1994), values are "a desirable trans situational goal varying in importance, which serves as a guiding principle in the life of a person or other social entity" (page 21). NEP, on the other hand, focuses on beliefs in the limit of growth and the need to preserve natural balance endangered by reckless development of human activities; it represents a widely adopted measure of pro-environmental orientation (Dunlap, Van Liere, Mertig, \& Jones, 2000). VBN suggests focusing on a chain of variables, from general pro-environmental values and concern to specific beliefs on the consequences of certain activities, and the responsibility of individuals to avoid such detrimental consequences: sustainable personal norms for pro-environmental behavior should be activated, guiding individuals towards greener behavioral patterns.

A third stream of research on pro-environmental behaviors is represented by habits (here analyzed outside of TPB-based frameworks), which assume particular relevance for mobility since behaviors are performed in stable contexts and decisional settings (Aarts \& Dijksterhuis, 2000; Verplanken, Aarts, van Knippenberg, \& van Knippenberg, 1994). According to the Theory of Interpersonal Behavior (Triandis, 1977), when individuals frequently perform a given behavior in response to a specific goal (like commuting to work, university or shopping) behavioral intentions no longer act as the main predictor of behavior itself. Habits hence represent an independent determinant of behavior (Bamberg \& Schmidt, 2003), moderating the intention-behavior relationship (Verplanken et al., 1998). Whereas habit has been sometimes used as a synonym of (or at least as a construct very close to) past behavior (Triandis, 1980), consistently with recent literature we consider the former as a more complex construct: habits represent goal-oriented scripts that are based on repeated behaviors and carried out in stable contexts (Ouellette \& Wood, 1998; Verplanken \& Aarts, 1999). We hence focus on past behaviors and habits as distinct predictors of given travel mode choices.

Albeit planned behavior, values and habits represent the three principal streams of research on travel modes, there are other variables that have been investigated in literature, and need to be taken into consideration. For instance, not only subjective (sometimes referred to as injunctive, or social) and personal (moral) norms but also descriptive norms can represent relevant predictors of intentions and behaviors. Descriptive norms represent typical and normal behaviors, what people do in a given situation (Cialdini, Kallgren, \& Reno, 1991; Cialdini, Reno, \& Kallgren, 1990): the perception of how people behave represents a motivation to do the same, providing "evidence as to what will likely be effective and adaptive action" (Cialdini et al., 1990, page 1015). Personal norms might be activated by problem awareness and by environmental values (Nordlund \& Garvill, 2003), which can also be considered as predictors of pro-environmental behaviors including mobility. Another example is represented by the Technology Acceptance Model (TAM, Davis, 1989), precursor of TRA and TPB, which suggests that perceived usefulness and perceived ease of use explain attitudes, which in turn explain behavioral intentions and actual behaviors.

Other theoretical frameworks stemming from the Model of Material Possession (Dittmar, 1992) focus on the functions that possessing specific goods such as private cars can fulfill. Instrumental motives have been object of most early research, as they relate to traditional dimensions such as convenience, speed or flexibility. Indeed, most research focused on examining the rational, instrumental benefits of private car use over public transport or other environment-friendly transport modes: empirical evidence suggested that most individuals tend to choose private cars as they believe it is faster, more accessible and reliable (Gatersleben, 2011). Recently researchers are explicitly studying also symbolic and affective motives, which relate to the identity of the self and the social position, and to emotions evoked by driving cars, respectively (Steg, 2005). These different motives can be linked to (and are the subject of) previously mentioned psychological theories. TPB for instance focuses on instrumental motives and on some specific social motives: most studies investigating attitudes focus on the instrumental consequences of car use, while subjective norms refer to the motivation to comply with expectations of reference groups, thus reflecting symbolic factors. Also other theories focused on social and symbolic motives, such as the Theory of Normative Conduct (Cialdini et al., 1991), Social Comparison Theory (Festinger, 1954; Masters \& Smith, 1987) and Self-presentation Theory (Schlenker, 1980), while some authors focus on the affect-driven dimension, suggesting that driving (or choosing different transport modes) evokes emotions that are anticipated in the decision process, thus determining modal choice (Manstead \& Parker, 1995).

\section{Methods}

The aim of this study is to synthesize empirical evidence on the determinants of travel mode choice, as regards both private car and environment-friendly alternatives: we label them as car and non-car (green), respectively. ${ }^{2}$

We first identified studies focusing on travel behavior (intention) using the internet search machine Google Scholar and the Web of Science, EBSCOhost, Scopus, and ScienceDirect databases. Search keywords were terms referring to travel modes,

\footnotetext{
${ }^{2}$ Green transport modes include public transport, bicycles, walking and reduction in (intention/ willingness to reduce) car use and carpooling.
} 
theoretical frameworks with respective determinants plus synonyms and/or combinations (see Appendix). After this preliminary search, 185 titles were selected as potential candidates for inclusion: abstracts and methodology sections were checked to identify whether studies were eligible for our research, leaving us with 73 studies. We read them and manually added 13 more articles adopting an ancestry approach. ${ }^{3}$ After a case-by-case discussion with an external expert in the field, ${ }^{4}$ who also replicated the search with relevant key-words for a further cross-check, we excluded 10 studies either because (i) they focus on the adoption of innovative technologies such as electric vehicles rather than on travel mode choice (e.g., Lai, Liu, Sun, Zhang, \& Xu, 2015), (ii) they address active transportation for leisure time or health-related issues, so that car is not an alternative (e.g., Lee \& Shepley, 2012), (iii) their sample consists of a very specific group hindering generalizability of findings (e.g., Murtagh, Rowe, Elliott, McMinn, \& Nelson, 2012). Lastly, 25 studies failing to report the quantitative information needed for the meta-analysis (bivariate Pearson correlations and sample sizes) were dropped. The final database (see Table 1 ) is hence based on 51 articles, 7 of which providing two independent samples datasets, for a total of 58 sample studies $(n=58)$.

As regards the meta-analytic strategy, we extracted 13 determinants of behavior and 13 determinants of intention from the selected studies. ${ }^{5}$ Based on the operational definitions of constructs, the determinants of travel mode choice are treated separately for car and non-car. We hence obtain four outcome (dependent) variables for the meta-analysis: car use behavior, non-car use behavior, car use intention and non-car use intention. For studies including more than one behavioral measure from the same sample, reporting separate bivariate correlations for each measure (e.g., Noblet et al., 2014; Steg and Sievers, 2000), the weighted average correlation of the behaviors within the study is used as a unit of analysis; this is done to follow the independence assumption underlying the validity of meta-analytic procedures.

For all computations and analyses, the statistical software Comprehensive Meta Analysis 3.3 was used. We first perform effect size analysis based on the correlation coefficients extracted from sample studies, pooling the effects from primary studies to assess the overall effect size of each independent variable on the dependent variables (Field, 2005). Following Hedges and Olkin (1985), we apply the Fisher's Z score transformation to calculate the weighted average correlation and to assign weights to individual effect sizes (for the specific formulas and a thorough description of the methodology, see Hedges and Olkin (1985) and Hedges and Vevea (1998)). Weights of individual effect sizes are assigned based upon the studies' sample sizes, with standard error and sample variance being calculated during the process; the significance of the effect size is measured by the Z-test and the precision of the pooled effect size is estimated by the 95\% confidence intervals.

Heterogeneity of results is tested through $I$-squared, which describes the percentage of variation across studies that is due to true differences in effect sizes rather than chance (Higgins, Thompson, Deeks, \& Altman, 2003). There is no clear consensus among researchers on the adequacy of fixed vs. random effect model for the effect size calculation (Field, 2005): while the former assumes a fixed weight for a specific study, the latter assumes that effect size varies randomly across studies. Consistently with most research in social sciences and following the argument of Hedges and Vevea (1998) about the inability of fixed effect methodology results to be generalized, we apply random effect methodology.

Moreover, robustness of findings against publication bias is assessed by calculating the fail-safe N, which represents the number of missing studies averaging a $Z$-value of zero that should be added to yield a statistically insignificant overall effect size (Rosenthal, 1984).

\section{Results and discussion}

Meta-analysis results are presented in Tables 2-5, illustrating the correlates of four outcome variables: behaviors and behavioral intentions as regards both private car use (car) and alternative, environment-friendly transport modes (green).

The first piece of information emerging from the analysis is represented by the combined effect size $\hat{\mathrm{r}}$, which according to rules of thumb in literature (Cohen, 1992) is considered large, medium and small at the $.50, .30$ and .10 marks, respectively.

Consistently with the TPB framework, according to which intentions are the main antecedents of behaviors and have in turn attitudes, norms and PBC as predictors, the meta-analysis confirms that indeed intentions ${ }^{6}$ represent the main predictor of travel mode choice. In literature, intentions and actual behaviors are sometimes collapsed in one single, overarching construct. Notwithstanding the overlapping and correlations between the two, however, we strongly suggest disentangling them: it is indeed of paramount importance to analyze in details the intention-behavior gap (Sheeran, 2002), and which drivers and predictors vary significantly in relevance according to a focus on either intentions or actual behaviors.

Besides intentions, habits and past use represent the main predictors, showing the highest correlations both with intentions and actual behaviors. Results corroborate speculations that especially in a domain characterized by stable context and settings such as commuting to work or to shopping (Aarts \& Dijksterhuis, 2000), there is a strong path dependency that heavily affects our mobility-related choices (Aarts, Verplanken, \& Knippenberg, 1998). This has relevant implications for policies

\footnotetext{
${ }^{3}$ An ancestry approach is a methodology widely adopted in literature reviews and meta-analyses in which the bibliographies and reference sections of studies already retrieved are used to locate earlier relevant studies (the "ancestors").

4 A University faculty member with specific expertise and international publications in meta-analyses in the domain of sustainability.

5 These determinants are attitude, injunctive (subjective/social) norm, descriptive norm, personal moral norm, perceived behavioral control, habit, past use, problem awareness, awareness of consequences, ascription of responsibility, environmental concern, environmental values, perceived usefulness, and intention (when behavior is the dependent variable). Personal and moral norms are merged as studies included in the analysis often use them interchangeably (Gardner \& Abraham, 2008).

${ }^{6}$ when analyzed as predictors, and not as outcome - dependent variables.
} 
Table 1

Characteristics of study dataset.

\begin{tabular}{|c|c|c|c|c|}
\hline Primary studies & Theory applied & Outcome variable(s) & Sample size & Country \\
\hline Abrahamse, Steg, Gifford, and Vlek (2009) & NAM, TPB & $\begin{array}{l}\text { Frequency of car trips, intention to reduce } \\
\text { car use }\end{array}$ & 241 & Canada \\
\hline Baldassare and Katz (1992) & $\mathrm{N} / \mathrm{A}$ & Frequency of reduced driving & 641 & USA \\
\hline Bamberg, Ajzen, and Schmidt (2003) & TPB, habit & Bus use; intention to use bus & 1874 & Germany \\
\hline Bamberg et al. (2007) & NAM, TPB & PT use; intention to use PT & 796,437 & Germany \\
\hline Carrus, Passafaro, and Bonnes (2008) & MGB & Intention to use PT & 180 & Italy \\
\hline Chen and Chao (2011) & TPB, TAM, Habit & Intention to use PT & 442 & Taiwan \\
\hline Cools et al. (2011) & TDM, NAM, VBN & $\begin{array}{l}\text { Willingness to reduce negative effects of } \\
\text { car use }\end{array}$ & 300 & Belgium \\
\hline $\begin{array}{l}\text { de Bruijn, Kremers, Schaalma, Van } \\
\text { Mechelen, and Brug (2005) }\end{array}$ & TPB & $\begin{array}{l}\text { Frequency of bicycle use; intention to use } \\
\text { bicycle }\end{array}$ & 3859 & Netherlands \\
\hline $\begin{array}{l}\text { de Bruijn, Kremers, Singh, Van den Putte, } \\
\text { and Van Mechelen (2009) }\end{array}$ & TPB & $\begin{array}{l}\text { Average cycling time; intention to use } \\
\text { bicycle }\end{array}$ & 317 & Netherlands \\
\hline De Groot and Steg $(2007)^{\mathrm{a}}$ & TPB(extended) & Intention to use transferium & 68,150 & Netherlands \\
\hline De Groot, Steg, and Dicke (2008) & NAM & Intention to reduce car use & 489 & 5 EU Countries \\
\hline Donald et al. (2014) & TPB (extended) & Car and PT use; intention to use car and PT & 827 & UK \\
\hline Eriksson and Forward (2011) & TPB & Intention to use car and other modes & 620 & Sweden \\
\hline Eriksson et al. (2006) $)^{a}$ & TDM, VBN & Willingness to reduce car use & 462,460 & Sweden \\
\hline Eriksson, Garvill, and Nordlund (2008) & VBN, Habit & Frequency of car trips & 71 & Sweden \\
\hline Forward (2014) & TPB, TTM, habit & Willingness to bike & 414 & Sweden \\
\hline $\begin{array}{l}\text { Friedrichsmeier, Matthies, and Klöckner } \\
\text { (2013) }\end{array}$ & Habit & $\%$ of car use; intention to use car & 1048 & Germany \\
\hline Fujii (2006) & TPB (extended) & Intention to reduce car use & 341 & Japan \\
\hline Gardner (2009) ${ }^{\mathrm{a}}$ & Habit, motivation & $\begin{array}{l}\% \text { of car } \& \text { bicycle trips; intention to use } \\
\text { car \& bicycle }\end{array}$ & 107,102 & UK, Netherlands \\
\hline Gardner and Abraham (2010) & TPB & $\begin{array}{l}\text { Proportion of car to non-car use; intention } \\
\text { to use car }\end{array}$ & 190 & UK \\
\hline Gärling et al. (2001)a & $\mathrm{N} / \mathrm{A}$ & $\begin{array}{l}\text { Car use frequency; car preference } \\
\text { (hypothetical scenario) }\end{array}$ & 60,48 & Sweden \\
\hline Garvill, Marell, and Nordlund (2003) & Attitude, Habit & Car use frequency & 115 & Sweden \\
\hline Harland et al. (1999) & TPB, NAM & Intention to use other modes than car & 305 & Netherlands \\
\hline Haustein and Hunecke (2007) & TPB (extended) & $\begin{array}{l}\% \text { of actual use; intention to use other } \\
\text { modes }\end{array}$ & 1545 & Germany \\
\hline Haustein, Klöckner, and Blöbaum (2009) & TPB, NAM & $\%$ of car use; intention to use PT & 2612 & Germany \\
\hline Heath and Gifford (2002) & TPB, NAM & $\begin{array}{l}\text { Percentage of bus use; intention to use } \\
\text { bus }\end{array}$ & 175 & Canada \\
\hline Hsiao and Yang (2010) & TPB (extended) & Willingness to take high speed rail & 300 & Taiwan \\
\hline $\begin{array}{l}\text { Joireman, Van Lange, Kuhlman, Van Vugt, } \\
\text { and Shelley (1997) }\end{array}$ & $\begin{array}{l}\text { Interdependence } \\
\text { theory }\end{array}$ & $\begin{array}{l}\text { Preference of car vs. other modes } \\
\text { (hypothetical scenario) }\end{array}$ & 102 & Netherlands \\
\hline Kaiser, Ranney, Hartig, and Bowler (1999) ${ }^{a}$ & $\begin{array}{l}\text { Rational choice, } \\
\text { NAM }\end{array}$ & $\begin{array}{l}\text { Intention for pro-environment travel } \\
\text { behavior }\end{array}$ & 436,488 & Switzerland, USA \\
\hline Klöckner and Matthies (2009) & NAM, TPB, habit & Ratio of car trips to all trips & 430 & Germany \\
\hline Lo et al. $(2016)^{a}$ & TPB, PN, habit & Frequency of car use; intention to use car & 452,386 & Netherlands \\
\hline Lois et al. (2015) & TPB, ТTM & Intention for cycle commuting & 595 & Spain \\
\hline Loukopoulos and Gärling (2005) & $\mathrm{N} / \mathrm{A}$ & Driving frequency; walking frequency & 155 & Sweden \\
\hline Mann and Abraham (2012) & TPB (extended) & Car and PT use; intention to use car and PT & 229 & UK \\
\hline Nilsson and Küller (2000) & Attitude & $\begin{array}{l}\text { Distance driven by car during the previous } \\
\text { year }\end{array}$ & 421 & Sweden \\
\hline Noblet et al. (2014) & TPB (extended) & $\begin{array}{l}\text { Attempts to drive less and use of } \\
\text { alternative modes }\end{array}$ & 1340 & USA \\
\hline Nordlund and Garvill (2002) & NAM & Pro-environmental travel behavior & 1414 & Sweden \\
\hline Nordlund and Garvill (2003) & NAM, VBN & Willingness to reduce car use & 1467 & Sweden \\
\hline Nordlund and Westin (2013) & TPB, VBN, NAM & Intention to use train & 1238 & Sweden \\
\hline Onwezen, Antonides, and Bartels (2013) & NAM, TPB & Frequency of bike and PT use & 617 & Netherlands \\
\hline Passafaro et al. (2014) & MGB & Desire to use bicycle & 387 & Italy \\
\hline Polk (2003) & TPB, habit & $\begin{array}{l}\text { Regular car use, willingness to reduce car } \\
\text { use }\end{array}$ & 1180 & Sweden \\
\hline Staats, Harland, and Wilke (2004) & TPB, habit & $\begin{array}{l}\text { Intention to use travel modes other than } \\
\text { car }\end{array}$ & 150 & Netherlands \\
\hline Steg $(2004,2005)$ & TPB, TNC & Percentage of car trips & 113 & Netherlands \\
\hline Steg and Sievers (2000) & Cultural theory & $\begin{array}{l}\text { Annual distance driven by car; proportion } \\
\text { of car vs. other modes }\end{array}$ & 269 & Netherlands \\
\hline Tanner (1999) & ITB & Frequency of car use & 153 & Switzerland \\
\hline Thøgersen (2006) & NAM, SDT, CMDT & Frequency of PT use & 810 & Denmark \\
\hline Van Vugt, Meertens, and Lange (1995) & $\begin{array}{l}\text { Interdependence } \\
\text { theory }\end{array}$ & $\begin{array}{l}\text { Preference of car vs. other modes } \\
\text { (hypothetical scenario) }\end{array}$ & 56 & Netherlands \\
\hline Verplanken et al. (1994) & Attitude, Habit & Frequency of car use & 199 & Netherlands \\
\hline Verplanken et al. (1998) & TPB, Habit & Ratio of car use to other modes; intention & 200 & Netherlands \\
\hline
\end{tabular}


Table 1 (continued)

\begin{tabular}{llll}
\hline Primary studies & Theory applied & Outcome variable(s) & Country \\
\hline $\begin{array}{l}\text { Yang-Wallentin, Schmidt, Davidov, and } \\
\text { Bamberg (2004) }\end{array}$ & TPB & Percentage of PT use; intention to use PT 912 \\
\hline
\end{tabular}

Note: CMDT = Cognitive moral development theory (Kohlberg, 1984), ITB= Ipsative theory of behavior (Frey, 1988), NAM= Norm activation model (Schwartz, 1977; Schwartz \& Howard, 1981), SDT = Self-determination theory (Deci \& Ryan, 1985), TAM = Technology acceptance model (Davis, 1989), TDM = Travel demand management measures, TNC = Theory of normative conduct (Cialdini et al., 1990, 1991), TPB = Theory of planned behavior (Ajzen, 1985, 1991), VBN = Value-belief-norm (Stern, 2000; Stern et al., 1999), MGB = Model of goal-directed behavior (Perugini \& Bagozzi, 2001), N/A=not available.

a Authors conducted two independent studies.

Table 2

Effect size analyses of correlates of car use.

\begin{tabular}{|c|c|c|c|c|c|c|c|}
\hline Variables & $\mathrm{K}$ & Sample & $\hat{\mathrm{r}}$ & Z-value & 95\% C.I. & & $\mathrm{I}^{2}$ \\
\hline ATT (car) & 15 & 4290 & 0.406 & $6.012^{* * * *}$ & 0.282 & 0.516 & 96.642 \\
\hline ATT (green) & 7 & 3283 & -0.358 & $-3.739^{* * *}$ & -0.516 & -0.176 & 96.673 \\
\hline INJ. N. (car) & 10 & 2866 & 0.229 & $3.598^{* * *}$ & 0.117 & 0.335 & 88.771 \\
\hline INJ. N (green) & 3 & 3681 & -0.153 & $-3.250^{* * *}$ & -0.243 & -0.061 & 71.444 \\
\hline DES. N. (car) & 6 & 2199 & 0.255 & $1.766^{*}$ & -0.029 & 0.500 & 97.681 \\
\hline PER. N. (car) & 3 & 1655 & 0.362 & $15.419^{* * * *}$ & 0.319 & 0.403 & 0.000 \\
\hline PER. N. (green) & 7 & 4222 & -0.262 & $-4.120^{* * *}$ & -0.376 & -0.140 & 87.424 \\
\hline PBC (car use) & 7 & 2399 & 0.27 & $2.832^{* * *}$ & 0.085 & 0.437 & 95.183 \\
\hline PBC (green) & 4 & 1092 & -0.429 & $-2.714^{* *}$ & -0.659 & -0.127 & 96.623 \\
\hline AWAR. CONS. & 2 & 671 & -0.130 & $-1.076^{\text {n.s. }}$ & -0.352 & 0.107 & 88.865 \\
\hline PROB. AWAR. & 8 & 5545 & -0.17 & $-4.237^{* * *}$ & -0.250 & -0.094 & 82.76 \\
\hline ASC. RESP. & 3 & 644 & -0.144 & $-1.037^{\text {n.s. }}$ & -0.397 & 0.129 & 91.632 \\
\hline ENV. CONC. & 4 & 2621 & -0.195 & $-2.975^{* * *}$ & -0.316 & -0.067 & 89.850 \\
\hline HABIT (car) & 17 & 8098 & 0.416 & $5.967^{* * *}$ & 0.289 & 0.529 & 97.111 \\
\hline Past car use & 6 & 1699 & 0.686 & $5.150^{* * *}$ & 0.478 & 0.821 & 96.683 \\
\hline INT. (car) & 8 & 3441 & 0.825 & $6.286^{* * * *}$ & 0.668 & 0.912 & 99.084 \\
\hline INT. (green) & 2 & 3300 & -0.511 & $-2.251^{* *}$ & -0.784 & -0.073 & 98.197 \\
\hline
\end{tabular}

$\mathrm{K}=$ number of outcomes from sample studies; $\hat{\mathrm{r}}=$ overall effect size; C.I. = confidence interval; $\mathrm{I}^{2}=\mathrm{I}$-squared.

ATT = attitudes; INJ. N. = injunctive norms; DES. N. = descriptive norms; PER. N. = personal norms; PBC= perceived behavioral control; AWAR. CONS. = awareness of consequences; PROB. AWAR. = problem awareness; ASC. RESP. = ascription of responsibility; ENV. CONC: = environmental concern; INT. = intention.

n.s. non-significant.

p $<0.01$.

** $\mathrm{p}<0.05$.

${ }^{*} \mathrm{p}<0.1$.

Table 3

Effect size analyses of correlates of non-car use.

\begin{tabular}{|c|c|c|c|c|c|c|c|}
\hline Variables & $\mathrm{K}$ & Sample & $\hat{\mathrm{r}}$ & Z-value & 95\% C.I. & & $\mathrm{I}^{2}$ \\
\hline ATT (green) & 12 & 13282 & 0.313 & $8.689^{* * * *}$ & 0.245 & 0.377 & 93.812 \\
\hline INJ. N. (green) & 12 & 12737 & 0.234 & $7.773^{* * * *}$ & 0.177 & 0.291 & 90.305 \\
\hline DES. N. (green) & 4 & 2231 & 0.214 & $2.375^{* *}$ & 0.038 & 0.377 & 93.553 \\
\hline PER. N. (green) & 9 & 6216 & 0.336 & $6.533^{* * *}$ & 0.24 & 0.425 & 93.983 \\
\hline PBC (green) & 12 & 12649 & 0.376 & $7.643^{* * *}$ & 0.286 & 0.460 & 96.687 \\
\hline AWAR. CONS. & 3 & 1571 & 0.125 & $1.729^{*}$ & -0.017 & 0.263 & 87.827 \\
\hline PROB. AWAR. & 5 & 2698 & 0.196 & $3.127^{* * * *}$ & 0.074 & 0.312 & 88.112 \\
\hline ASC. RESP. & 4 & 1746 & 0.223 & $4.051^{* * *}$ & 0.122 & 0.339 & 82.018 \\
\hline ENV. CONC. & 3 & 936 & 0.139 & $4.563^{* * *}$ & 0.079 & 0.197 & 59.635 \\
\hline HABIT (green) & 2 & 929 & 0.683 & $2.005^{* *}$ & 0.019 & 0.929 & 98.367 \\
\hline Past non-car use & 3 & 2205 & 0.846 & $5.741^{* * * *}$ & 0.674 & 0.931 & 97.493 \\
\hline ENV. VAL. & 4 & 4417 & 0.140 & $1.456^{\text {n.s. }}$ & -0.049 & 0.319 & 97.298 \\
\hline INT (green) & 12 & 11411 & 0.617 & $7.308^{* * * *}$ & 0.484 & 0.723 & 98.977 \\
\hline
\end{tabular}

$\mathrm{K}=$ number of outcomes from sample studies; $\hat{\mathrm{r}}=$ overall effect size; C.I. = confidence interval; $\mathrm{I}^{2}=\mathrm{I}$-squared.

ATT = attitudes; INJ. N. = injunctive norms; DES. N. = descriptive norms; PER. N. = personal norms; PBC= perceived behavioral control; AWAR. CONS.

= awareness of consequences; PROB. AWAR. = problem awareness; ASC. RESP. = ascription of responsibility; ENV. CONC: = environmental concern; ENV.

VAL. = environmental values; INT. = intention.

n.s. non-significant.

${ }^{* * *} \mathrm{p}<0.01$.

** $\mathrm{p}<0.05$.

* $\mathrm{p}<0.1$. 
Table 4

Effect size analyses of correlates of intention to use car.

\begin{tabular}{|c|c|c|c|c|c|c|c|}
\hline Variables & $\mathrm{K}$ & Sample & $\hat{\mathrm{r}}$ & Z-value & $95 \% \mathrm{CI}$ & & $\mathrm{I}^{2}$ \\
\hline ATT (car) & 7 & 2906 & 0.563 & $5.916^{* * * *}$ & 0.402 & 0.690 & 96.832 \\
\hline ATT (green) & 4 & 1483 & -0.530 & $-4.021^{* * *}$ & -0.705 & -0.294 & 96.743 \\
\hline INJ. N. (car) & 7 & 2906 & 0.424 & $7.749^{* * * *}$ & 0.326 & 0.513 & 89.066 \\
\hline DES. N. (car) & 6 & 2706 & 0.272 & $1.968^{* *}$ & 0.001 & 0.506 & 98.048 \\
\hline PER. N. (car) & 3 & 1665 & 0.394 & $16.953^{* * *}$ & 0.353 & 0.434 & 0.000 \\
\hline PER. N. (green) & 2 & 421 & -0.512 & $-11.520^{* * *}$ & -0.580 & -0.438 & 0.000 \\
\hline $\mathrm{PBC}$ (car) & 7 & 2906 & 0.322 & $3.088^{* * * *}$ & 0.121 & 0.498 & 96.867 \\
\hline PBC (green) & 2 & 421 & -0.452 & $-4.077^{* * *}$ & -0.617 & -0.247 & 82.950 \\
\hline ENV. CONC. & 3 & 1103 & -0.259 & $-8.434^{* * *}$ & -0.315 & -0.201 & 0.000 \\
\hline HABIT (car) & 7 & 4068 & 0.472 & $7.195^{* * * *}$ & 0.357 & 0.573 & 94.612 \\
\hline Past car use & 4 & 1584 & 0.739 & $3.471^{* * * *}$ & 0.391 & 0.902 & 98.762 \\
\hline
\end{tabular}

$\mathrm{K}=$ number of outcomes from sample studies; $\hat{\mathrm{r}}=$ overall effect size; $\mathrm{C} . \mathrm{I}$. = confidence interval; $\mathrm{I}^{2}=\mathrm{I}$-squared.

ATT = attitudes; INJ. N. = injunctive norms; DES. N. = descriptive norms; PER. N. = personal norms; PBC= perceived behavioral control; AWAR. CONS. = awareness of consequences; PROB. AWAR. = problem awareness; ASC. RESP. = ascription of responsibility; ENV. CONC: = environmental concern; ENV. VAL. = environmental values; INT. $=$ intention.

n.s., non-significant.

" $\mathrm{p}<0.1$.

$\mathrm{p}<0.01$.

p** $<0.05$.

Table 5

Effect size analyses of correlates of intention to use non-car.

\begin{tabular}{|c|c|c|c|c|c|c|c|}
\hline Variables & $\mathrm{K}$ & Sample & $\hat{\mathrm{r}}$ & Z-value & $95 \%$ CI & & $\mathrm{I}^{2}$ \\
\hline ATT (car) & 4 & 4204 & -0.240 & $-2.796^{* * *}$ & -0.393 & -0.073 & 96.572 \\
\hline ATT (green) & 23 & 17824 & 0.467 & $11.086^{* * *}$ & 0.394 & 0.534 & 97.064 \\
\hline INJ. N. (car) & 2 & 391 & 0.255 & $1.936^{*}$ & -0.003 & 0.481 & 84.828 \\
\hline INJ. N. (green) & 20 & 16770 & 0.410 & $12.819^{* * *}$ & 0.353 & 0.464 & 94.061 \\
\hline DES. N. (green) & 7 & 3272 & 0.347 & $5.280^{* * *}$ & 0.224 & 0.459 & 93.095 \\
\hline PER. N. (green) & 13 & 8968 & 0.508 & $9.925^{* * *}$ & 0.421 & 0.585 & 95.996 \\
\hline PBC (green) & 23 & 15355 & 0.526 & $9.579^{* * *}$ & 0.434 & 0.607 & 98.083 \\
\hline AWAR. CONS. & 4 & 1684 & 0.236 & $3.213^{* * *}$ & 0.094 & 0.369 & 89.019 \\
\hline PROB. AWAR. & 14 & 13213 & 0.315 & $10.307^{* * *}$ & 0.258 & 0.370 & 91.603 \\
\hline ASC. RESP. & 7 & 2614 & 0.344 & $7.014^{* * *}$ & 0.253 & 0.429 & 84.746 \\
\hline ENV. CONC. & 14 & 5518 & 0.225 & $7.756^{* * *}$ & 0.170 & 0.280 & 72.609 \\
\hline HABIT (car) & 3 & 3818 & -0.096 & $-0.180^{\text {n.s. }}$ & -0.815 & 0.739 & 99.817 \\
\hline HABIT (green) & 4 & 1438 & 0.554 & $3.454^{* * *}$ & 0.264 & 0.752 & 97.144 \\
\hline Past non-car use & 6 & 3077 & 0.731 & $8.891^{* * *}$ & 0.620 & 0.813 & 95.686 \\
\hline ENV. VAL. & 9 & 7547 & 0.153 & $4.407^{* * *}$ & 0.086 & 0.220 & 88.016 \\
\hline PERC. USE. (green) & 2 & 671 & 0.421 & $11.580^{* * *}$ & 0.357 & 0.482 & 0.000 \\
\hline
\end{tabular}

$\mathrm{K}=$ number of outcomes from sample studies; $\hat{\mathrm{r}}=$ overall effect size; C.I. = confidence interval; $\mathrm{I}^{2}=\mathrm{I}$-squared.

ATT = attitudes; INJ. N. = injunctive norms; DES. N. = descriptive norms; PER. N. = personal norms; PBC= perceived behavioral control; AWAR. CONS

= awareness of consequences; PROB. AWAR. = problem awareness; ASC. RESP. = ascription of responsibility; ENV. CONC. = environmental concern; ENV.

VAL. = environmental values; PERC. USE. = perceived usefulness.

n.s., non-significant.

${ }^{* *} \mathrm{p}<0.05$.

$\mathrm{p}<0.01$

$\mathrm{p}<0.1$.

aiming at disrupting old, long-established behavioral patterns as to promote a shift towards innovative and more sustainable routines. According to the Habit Discontinuity Hypothesis (Verplanken \& Wood, 2006), when the context changes disrupting our habits a window opens, so that behaviors are more likely to be considered deliberately and alternatives rationally evaluated. As a consequence, "interventions may be more effective when these are delivered in association with a disruption of a stable context” (Verplanken, Walker, Davis, \& Jurasek, 2008, page 126): for instance, when we move to a new neighborhood, change job or face other events that modify our travel routines. Such life events evoke conscious reasoning and reorient cognitive gears back to deliberateness, being hence able to weaken car choice habit (Klöckner, 2004) and to induce a behavioral change towards sustainable mobility (Lanzendorf, 2010; Prillwitz, Harms, \& Lanzendorf, 2006). There is indeed an emerging stream of literature in transportation research that emphasizes the role of individuals' key life-course events and experiences in leading to change in travel behavior, referred as mobility biographies approach (Lanzendorf, 2003; Scheiner, 2007): such life events can be represented for instance by residential relocation and associated changes in the built environment (Scheiner \& Holz-Rau, 2013) but also by acquisition of driver's license, education status change, employment change (Klöckner, 2004; Van der Waerden, Borgers, \& Timmermans, 2003) or even first child birth, divorce and retirement (Schoenduwe, Mueller, Peters, \& Lanzendorf, 2015). 
Indeed, behaviors are the result of a trade-off between deliberate and non-deliberate factors (Van Acker, Van Wee, \& Witlox, 2010). The first time an individual adopts a deliberate behavior, as the outcome of a choice between feasible alternatives, he applies a rational thinking moderated by behavioral determinants (e.g., beliefs, perceptions, attitude, social influence etc.). Once the new behavior becomes routine and is repeated over and over in a given situation, deliberate factors don't seem to play their direct role any more: that is, later behavior is largely influenced by past behavior, and is no longer the outcome of a choice between the original alternatives. Consistently with the Theory of Repeated Behavior (Ronis, Yates, \& Kirscht, 1989), the joint effect of costs of learning and uncertainties about the impact of a different-from-past decision pushes individuals to "reuse past solutions to make their behavior easier and less risky. This mechanism is enhanced when individuals are constrained by time, budget, or social commitments." (Gärling \& Axhausen, 2003, page 2). As long as the given conditions remain stable, behavior would not change and would not necessarily represent the outcome of a deliberate choice between alternatives as it used to be in the beginning. On the other hand, as circumstances change (for instance, change in biographies, new policy interventions, unusual weather change, etc.) the process (might) restart from the beginning: that is, the behavior is once again consciously chosen. Daily commuting represents a typical situation of decisions taken in stable settings: as such, travel behaviors are seldom the outcome of an evaluation-based decision process, while they are highly affected by past behavior and habit.

Also the three planned behavior constructs (attitudes, norms and PBC) proved to have a good predictive capability and, consistently with prior research (Bamberg \& Möser, 2007; Gardner \& Abraham, 2008), they all appear to be closer to intentions than behaviors. Moreover, while attitudes and PBC seem the main predictors within this framework as regards both intentions and behaviors (Gardner \& Abraham, 2008), injunctive-subjective norms emerge as good predictors of behavioral intentions, only. This result contradicts Sheppard, Hartwick, and Warshaw (1988), according to whom subjective norms are indeed a weak predictor of intentions, as well.

All environmental variables directly connected with sustainability issues (environmental values, concern, etc.) seem to play a marginal role as regards the capability of predicting actual travel mode choices, while they emerge as significant predictors of intentions to choose an eco-friendly alternative. Heath and Gifford (2002) reported similar associations between environmental constructs and behavioral outcomes indicating a mediating role of intention. This is consistent with prior literature (Kennedy, Beckley, McFarlane, \& Nadeau, 2009) suggesting that many individuals fail to walk the talk: no matter how strong their environmental beliefs and awareness, they will fail to act accordingly to such pro-environmental profile. This is clearly problematic for policy makers and other actors aiming at modifying behavioral patterns of citizens, as initiatives aimed at increasing the awareness and the environmentalism of a community might fail in the end to lead to a concrete, effective behavioral shift. Further insights on the determinants of behavioral intentions and actual behaviors are needed, for instance, to understand which type of instrument (e.g., financial vs. non-financial appeal and inducements) prove to be more effective (Bolderdijk, Steg, Geller, Lehman, \& Postmes, 2013; Lanzini \& Thøgersen, 2014).

Robustness of results is supported by publication bias analysis, ${ }^{7}$ which suggests that in almost all cases such bias is absent: the only exceptions refer to awareness of consequences and ascription of responsibility as correlates of car use (Fail-safe $\mathrm{N}=5$ and 4, respectively), and injunctive norms towards car use as correlates of intentions to use alternative transport modes (Failsafe $\mathrm{N}=16$ ).

A crucial element to highlight is represented by the great heterogeneity of results, as suggested by the I-squared that in most cases is well above the 75\% threshold identifying a large heterogeneity (Higgins et al., 2003). Better understanding of the reasons underpinning such variability is required to set directions for future research as well as for practical decision making purposes (Möser \& Bamberg, 2008). We hence identify five study characteristics as potential moderators of the effect size distribution that could explain such heterogeneity, and we perform a moderator analysis, accordingly. The first moderator refers to the operationalization and measurement (MST) of behaviors (and intentions). Actual behaviors are measured with reference to a specific time-frame (e.g. "how many times did you drive a car over the past week?"), while typical behaviors are measured with no such reference (e.g. "how often do you drive a car?"); we classify measurement and operationalization into typical, actual-days (with reference to a time-frame of a week or less) and actual-weeks (with reference to a time-frame longer than a week). Based on the speculation that the purpose of the trip (TRIP) might affect which predictors assume a prominent role, we adopt a partition where trips have been classified, according to their specific purpose, into working trips, shopping trips, general trips and a residual category other trips. The study sample (SAMPLE) moderator is categorized into general population, students, employees, and others, as different groups might differ in terms of travel modes. Also geographical location (LOC) of the study (categorized into Europe, North America and Far East) and study period (YEAR) have been included as moderators in the analysis. Since each covariate is required to appear in at least 10 independent studies to be included in the analysis (Borenstein, Hedges, Higgins, \& Rothstein, 2009), we combine driving and non-driving intention and behavior datasets as to ensure the inclusion of all covariates in the analysis. To analyze the individual effect of each moderator we used random effects univariate meta-regression with Method of Moments (MM) estimation technique.

Results of heterogeneity analysis are presented in Table 6. Only cases where at least one predictor proved to be significant are reported; however, the complete set of calculations of sub-group and meta-regression analyses can be retrieved from the authors.

${ }^{7}$ For reasons of space only relevant results are presented, but the whole table can be retrieved from the authors. 
Table 6

Results of heterogeneity analysis.

\begin{tabular}{|c|c|c|c|c|c|c|}
\hline & & MST & TRIP & LOC & YEAR & SAMPLE \\
\hline Dependent Variables (ES) & $k$ & Q-stats (df) $R^{2}$ & Q-stats (df) $R^{2}$ & Q-stats(df) $R^{2}$ & Q-stats (df) $R^{2}$ & Q-stats (df) $R^{2}$ \\
\hline INT-BEH & 22 & $\begin{array}{l}30.3(2)^{* * * *} \\
63 \%\end{array}$ & $\begin{array}{l}9.85(2)^{* * *} \\
14 \%\end{array}$ & n.s. & $\begin{array}{l}15.0(1)^{* * * *} \\
34 \%\end{array}$ & $\begin{array}{l}11.2(2)^{* * * *} \\
25 \%\end{array}$ \\
\hline ATT-BEH & 37 & $\begin{array}{l}9.98(2)^{* * * *} \\
23 \%\end{array}$ & n.s. & n.s. & $\begin{array}{l}4.39(1)^{* * *} \\
1 \%\end{array}$ & $\begin{array}{l}22.01(2)^{* * * *} \\
27 \%\end{array}$ \\
\hline ATT-INT $^{\mathrm{a}}$ & 38 & $\begin{array}{l}4.69(2)^{*} \\
27 \%\end{array}$ & n.s. & n.s. & n.s. & $\begin{array}{l}7.87(3)^{* *} \\
27 \%\end{array}$ \\
\hline INJ-BEH & 28 & $\begin{array}{l}13.15(2)^{* * *} \\
18 \%\end{array}$ & n.s. & n.s. & n.s. & n.s. \\
\hline INJ-INT & 29 & n.s. & n.s. & n.s. & n.s. & $\begin{array}{l}7.96(3)^{* * *} \\
0 \%\end{array}$ \\
\hline DES-BEH & 10 & $\begin{array}{l}16.23(2)^{* * *} \\
50 \%\end{array}$ & $\begin{array}{l}13.2(1)^{* * * *} \\
54 \%\end{array}$ & n.s. & n.s. & n.s. \\
\hline DES-INT & 13 & $\begin{array}{l}33.0(2)^{* * * *} \\
62 \%\end{array}$ & $\begin{array}{l}4.43(1)^{* * *} \\
14 \%\end{array}$ & n.s. & n.s. & n.s. \\
\hline PER-BEH & 19 & n.s. & n.s. & n.s. & n.s. & $\begin{array}{l}6.96(2)^{* *} \\
15 \%\end{array}$ \\
\hline PBC-BEH & 26 & $\begin{array}{l}7.67(2)^{* *} \\
20 \%\end{array}$ & n.s. & n.s. & n.s. & n.s. \\
\hline PBC-INT & 32 & $\begin{array}{l}6.75(2)^{* *} \\
0 \%\end{array}$ & n.s. & n.s. & n.s. & n.s. \\
\hline ENV-BEH & 10 & $\begin{array}{l}7.48(2)^{* * * *} \\
40 \%\end{array}$ & $\begin{array}{l}12.6(1)^{* * *} \\
63 \%\end{array}$ & n.s. & n.s. & $\mathrm{N} / \mathrm{A}$ \\
\hline ENV-INT & 16 & n.s. & n.s. & n.s. & $\begin{array}{l}5.62(1)^{* * *} \\
15 \%\end{array}$ & n.s. \\
\hline PAST-INT & 10 & $\begin{array}{l}10.2(2)^{* *} \\
12 \%\end{array}$ & n.s. & $\mathrm{N} / \mathrm{A}$ & n.s. & $\begin{array}{l}25.0(3)^{* * *} \\
55 \%\end{array}$ \\
\hline
\end{tabular}

Note: Q-stat = chi-square distribution with $\mathrm{n}-1$ degrees of freedom $(\mathrm{df})$, where $\mathrm{n}$ is the number of predictors in the model. It tests whether at least one of the regression coefficients in the model is different from zero; a significant $\mathrm{Q}$-stat confirms the relevance of covariates to the predicted effect size. $\mathrm{R}^{2}=$ the proportion of true variance explained by the model.

ES = effect size; $\mathrm{BEH}=$ behavior; $\mathrm{INT}=$ intention; $\mathrm{INJ}=$ injunctive-subjective norms; $\mathrm{DES}=$ descriptive norms; $\mathrm{PER}=$ personal norms; PBC = perceived behavioral control; PAST $=$ past use .

n.s., non-significant.

p $<0.01$.

*** $\mathrm{p}<0.05$.

$\mathrm{p}<0.1$.

a P-value $<0.05$ is the decision rule for the significance of the relationships. However, only in two cases, when $\mathrm{R}^{2}$ is high, $10 \%$ significance level is considered.

The analysis shows that measurement is the prominent factor affecting heterogeneity of results: methodological aspects of surveys such as the operationalization and measurement of behaviors and intentions heavily affect the outcome in terms of correlation with relevant predictors. This has evident implications for policy makers. Indeed, since most Travel Demand Management (Eriksson, Garvill, \& Nordlund, 2006) and soft transport policy (Möser \& Bamberg, 2008) measures have their roots in the informational background provided by analyses of commuters' decision-making processes, it is necessary to better understand the relationship between the operationalization and measurement of constructs and the results of empirical investigations. This will prevent policy makers from shaping strategies based on an over-simplified interpretation of the information at hand.

Also trip purpose and sample type explain heterogeneity of results, though to a lesser extent compared to measurement. As regards sample type, some groups (e.g., students) display specific features that affect their behavioral patterns and the respective predictors, albeit there is evidence that socio-demographics are not effective determinants of proenvironmental behaviors (Diamantopoulos, Schlegelmilch, Sinkovics, \& Bohlen, 2003). Consistently with our results, trip purpose has been investigated in literature as a variable shaping modal choice (De Witte, Hollevoet, Dobruszkes, Hubert, \& Macharis, 2013). For instance, there is evidence that while car use in prominent for business (Limtanakool, Dijst, \& Schwanen, 2006; O'Fallon et al., 2004) or shopping (Kim \& Ulfarsson, 2008) trips, alternative modes are more frequents for school (Kim \& Ulfarsson, 2008) or short social (Pucher \& Renne, 2003) trips.

The low moderating effect of study period is somehow surprising, as it contradicts speculations that over the past 25 years sustainability gained unprecedented relevance in shaping behavioral patterns (Akehurst, Afonso, \& Gonçalves, 2012). On the other hand, policy makers can benefit from such stability, as an ever-changing context would represent a hindering factor for the setting up and the implementation of sustainable mobility strategies.

Location is the only mediator analysed that does not explain heterogeneity in effect sizes. This might look surprising, given the differences between the countries where primary studies were performed and the subsequent assumption that both cultural and contextual factors could affect the heterogeneity of results. However, it is worth noting that results are 
consistent with evidence suggesting that psychological determinants of TPB are generally homogeneous across different regions (Lo, van Breukelen, Peters, \& Kok, 2016). With regard to location, one possible speculation that should be addressed by future research is whether other variables might have better explanatory power compared to the country or the macroregion where the studies are performed. For instance, it might be interesting to investigate more in detail the specific features of the area where data are collected: is it a rural or a metropolitan area? Does it have an efficient network of public transportation? Is there an effective involvement of public authorities for the planning and implementation of sustainable mobility plans?

\section{Conclusions}

Increased car dependency represents a crucial challenge of our times (Blythe, 2005), given the economic, environmental and societal repercussions of private mobility (Schuitema, Steg, \& Forward, 2010). Different strategies have been hence proposed to lower the ecological footprint of current travel patterns and to shape new, more sustainable mobility-related behaviors. Given the inconsistent results of literature on transport mode choice and the awareness of the need to gain better understanding of socio-cognitive factors affecting such choice (Cools et al., 2011; Eriksson et al., 2006), we run a metaanalysis to synthesize available evidence, investigating the psychological and behavioral correlates of both private car use and alternative, environment-friendly transport modes. We build on the work of Gardner and Abraham (2008), including recent studies and broadening the analysis encompassing new predictors and perspectives; moreover, we run heterogeneity analysis to explain the variability of results.

Our work has relevant implications especially for policy makers, willing to implement sound mobility plans that require the essential contribution of individual behaviors (De Witte et al., 2013). Some general patterns clearly emerge from the analysis, such as the predominance of intentions, habits and past behavior as predictors of travel mode choice or the intention-behavior gap. However, policy makers should be careful in interpreting such an informational background, avoiding a simplistic and superficial approach that would hinder the effectiveness of policies: for instance, the methodological heterogeneity of primary studies (e.g., the measurement and operationalization of constructs) represents a problematic aspect, as the framing of survey questions has a relevant impact on the outcome on which policy makers are supposed to base their strategies. Whereas a homogenization of methodological frameworks would be particularly complex on a practical standpoint, policy makers or other actors interpreting available information should put extra care in focusing not only on the final results of the analysis, but also on the study characteristics that led to such results.

From the point of view of research, future investigations could first of all increase the number of studies analyzing scantly investigated predictors (that, given the low number of observations, could not be included in our meta-analysis). Moreover, they could as anticipated be more specific in reporting relevant aspects (both contextual and methodological) of the study, specifying for instance the features of both the area and the community where data are collected, as this might represent a factor capable of explaining part of the variability in results. Thirdly, future research should analyze more in detail the effects that some variables exert on (and interacting with) constructs at the base of the theoretical frameworks examined. This is the case, for instance, of so-called residential self-selection and its effects on PBC: indeed, in the domain of mobility self-selection refers to the tendency of people to choose locations based on their travel abilities, needs and preferences (Litman, 2005). The inclusion of a further level of analysis might increase the complexity of the model; yet, such approach could shed further light on the real motives underpinning modal choice, and different methodologies have been proposed in literature to address endogeneity biases (Mokhtarian \& Cao, 2008; Winship \& Morgan, 1999).

The need to gain further insights on the determinants of modal choices is urgent; albeit the intertwining effect of a wide range of variables (both subjective and contextual) makes the path long and complex, this is no good reason to give up on the task as the reward is well worth the effort.

\section{Appendix A. Search keywords}

In the search of relevant literature, we used the following words (plus synonyms and combinations):

Alternative travel modes

Altruistic value

Ascription of responsibility

Attitudes

Awareness of consequences

Bicycle use

Biospheric value

Bus use

Car (use) intention

Car behavior

Car sharing

Car use

Carpooling 
Descriptive norms

Driving behavior

Ease of use

Egoistic value

Environmental belief

Environmental concern

Environmental value

General values

Guilt

Habit

Habit strength

Injunctive norms

Mobility

Moral norms

New Ecological Paradigm (and NEP)

Non-car (use) intention

Non-car use

Norm activation

Past behavior

Past use

Peak car use

Perceived behavioral control

Perceived usefulness

Personal car use

Personal norms

Personal values

Private car use

Pro-environmental mobility

Pro-environmental travel behavior

Problem awareness

Public transport

Public transport intention

Reduce car use

Response frequency measure

Self reported habit index (and SRHI)

Self-efficacy

Self-enhancement value

Self-transcendence value

Social norms

Subjective norms

Sustainable mobility

Sustainable travel behavior

Sustainable travel intention

Technology acceptance model (and TAM)

Theory of planned behavior (and TPB)

Theory of reasoned action (and TRA)

Train use

Travel behavior

Travel mode choice

Value belief norm (and VBN)

Value orientation

Walking

Willingness to reduce car

\section{References}

Aarts, H., \& Dijksterhuis, A. (2000). Habits as knowledge structures: Automaticity in goal-directed behavior. Journal of Personality and Social Psychology, 78 (1), 53-63. http://dx.doi.org/10.1037/0022-3514.78.1.53.

Aarts, H., Verplanken, B., \& Knippenberg, A. (1998). Predicting behavior from actions in the past: Repeated decision making or a matter of habit? Journal of Applied Social Psychology, 28(15), 1355-1374. http://dx.doi.org/10.1111/j.1559-1816.1998.tb01681.x. 
Abrahamse, W., Steg, L., Gifford, R., \& Vlek, C. (2009). Factors influencing car use for commuting and the intention to reduce it: A question of self-interest or morality? Transportation Research Part F: Traffic Psychology and Behaviour, 12(4), 317-324. http://dx.doi.org/10.1016/j.trf.2009.04.004.

Ajzen, I. (1991). The theory of planned behavior. Organizational Behavior and Human Decision Processes, 50(2), 179-211. http://dx.doi.org/10.1016/07495978(91)90020-T.

Ajzen, I. (1985). From intentions to actions: A theory of planned behavior. In J. Kuhl \& J. Beckmann (Eds.), Action-control: From cognition to behavior (pp. 11-39). Heidelberg: Springer.

Akehurst, G., Afonso, C., \& Gonçalves, H. M. (2012). Re-examining green purchase behaviour and the green consumer profile: New evidences. Management Decision, 50(5), 972-988. http://dx.doi.org/10.1108/00251741211227726.

Altenburg, T., Schamp, E. W., \& Chaudhary, A. (2015). The emergence of electromobility: Comparing technological pathways in France, Germany, China and India. Science and Public Policy (October) (pp. 1-12), http://dx.doi.org/10.1093/scipol/scv054.

Armitage, C. J., \& Conner, M. (2001). Efficacy of the theory of planned behaviour: A meta-analytic review. British Journal of Social Psychology, 40(4), 471-499. http://dx.doi.org/10.1348/014466601164939.

Baldassare, M., \& Katz, C. (1992). The personal threat of environmental problems as predictor of environmental practices. Environment and Behavior, 24(5), 602-616. http://dx.doi.org/10.1177/0013916592245002.

Bamberg, S., Ajzen, I., \& Schmidt, P. (2003). Choice of travel mode in the theory of planned behavior: The roles of past behavior, habit, and reasoned action. Basic and Applied Social Psychology, 25(3), 175-187. http://dx.doi.org/10.1207/S15324834BASP2503_01.

Bamberg, S., Hunecke, M., \& Blöbaum, A. (2007). Social context, personal norms and the use of public transportation: Two field studies. Journal of Environmental Psychology, 27(3), 190-203. http://dx.doi.org/10.1016/j.jenvp.2007.04.001.

Bamberg, S., \& Möser, G. (2007). Twenty years after Hines, Hungerford, and Tomera: A new meta-analysis of psycho-social determinants of proenvironmental behaviour. Journal of Environmental Psychology, 27(1), 14-25. http://dx.doi.org/10.1016/j.jenvp.2006.12.002.

Bamberg, S., \& Schmidt, P. (2003). Incentives, morality, or habit? Predicting students' car use for university routes with the models of Ajzen, Schwartz, and Triandis. Environment and Behavior, 35(2), 264-285. http://dx.doi.org/10.1177/0013916502250134.

Blythe, P. T. (2005). Congestion charging: Technical options for the delivery of future UK policy. Transportation Research Part A: Policy and Practice, 39(7), 571-587. http://dx.doi.org/10.1016/j.tra.2005.02.012.

Bolderdijk, J. W., Steg, L., Geller, E. S., Lehman, P. K., \& Postmes, T. (2013). Comparing the effectiveness of monetary versus moral motives in environmental campaigning. Nature Climate Change, 3(4), 413-416. http://dx.doi.org/10.1038/nclimate1767.

Borenstein, M., Hedges, L. V., Higgins, J. P. T., \& Rothstein, H. R. (2009). Introduction to meta-analysis. Chichester, England: Wiley.

CAAM (2015). China Automotive Industry Yearbook 2000-2014. Beijing: China Association of Automobile Manufacturers. Retrieved from <http://www.caam. org.cn/english/>.

Carrus, G., Passafaro, P., \& Bonnes, M. (2008). Emotions, habits and rational choices in ecological behaviours: The case of recycling and use of public transportation. Journal of Environmental Psychology, 28(1), 51-62. http://dx.doi.org/10.1016/j.jenvp.2007.09.003.

Chen, C. F., \& Chao, W. H. (2011). Habitual or reasoned? Using the theory of planned behavior, technology acceptance model, and habit to examine switching intentions toward public transit. Transportation Research Part F: Traffic Psychology and Behaviour, 14(2), 128-137. http://dx.doi.org/10.1016/j. trf.2010.11.006.

Cialdini, R. B., Kallgren, C. A., \& Reno, R. R. (1991). A focus theory of normative conduct: A theoretical refinement and reevaluation of the role of norms in human behavior. Advances in Experimental Social Psychology, 24, 201-234. http://dx.doi.org/10.1016/S0065-2601(08)60330-5.

Cialdini, R. B., Reno, R. R., \& Kallgren, C. A. (1990). A focus theory of normative conduct: Recycling the concept of norms to reduce littering in public places. Journal of Personality and Social Psychology, 58(6), 1015-1026. http://dx.doi.org/10.1037/0022-3514.58.6.1015.

Cohen, J. (1992). A power primer. Psychological Bulletin, 112(1), 155-159. http://dx.doi.org/10.1037/0033-2909.112.1.155.

Collins, C. M., \& Chambers, S. M. (2005). Psychological and situational influences on commuter-transport-mode choice. Environment and Behavior, 37(5), 640-661. http://dx.doi.org/10.1177/0013916504265440.

Conner, M., \& Armitage, C. J. (1998). Extending the theory of planned behavior: A review and avenues for further research. Journal of Applied Social Psychology, 28(15), 1429-1464. http://dx.doi.org/10.1111/j.1559-1816.1998.tb01685.x.

Cools, M., Brijs, K., Tormans, H., Moons, E., Janssens, D., \& Wets, G. (2011). The socio-cognitive links between road pricing acceptability and changes in travelbehavior. Transportation Research Part A: Policy and Practice, 45(8), 779-788. http://dx.doi.org/10.1016/j.tra.2011.06.006.

Davis, F. D. (1989). Perceived usefulness, perceived ease of use, and user acceptance of information technology. MIS Quarterly, 13(3), 319-340. http://dx.doi. org/10.2307/249008.

de Bruijn, G. J., Kremers, S. P., Schaalma, H., Van Mechelen, W., \& Brug, J. (2005). Determinants of adolescent bicycle use for transportation and snacking behavior. Preventive Medicine, 40(6), 658-667. http://dx.doi.org/10.1016/j.ypmed.2004.09.003.

de Bruijn, G. J., Kremers, S. P., Singh, A., Van den Putte, B., \& Van Mechelen, W. (2009). Adult active transportation: Adding habit strength to the theory of planned behavior. American Journal of Preventive Medicine, 36(3), 189-194. http://dx.doi.org/10.1016/j.amepre.2008.10.019.

De Groot, J., \& Steg, L. (2007). General beliefs and the theory of planned behavior: The role of environmental concerns in the TPB. Journal of Applied Social Psychology, 37(8), 1817-1836. http://dx.doi.org/10.1111/j.1559-1816.2007.00239.x.

De Groot, J. I. M., Steg, L., \& Dicke, M. (2008). Transportation trends from a moral perspective: Value orientations, norms and reducing car use. In F. N. Gustavsson (Ed.), New transportation research progress (pp. 67-91). Hauppauge, NY: Nova Science Publishers.

De Witte, A., Hollevoet, J., Dobruszkes, F., Hubert, M., \& Macharis, C. (2013). Linking modal choice to motility: A comprehensive review. Transportation Research Part A: Policy and Practice, 49(March), 329-341. http://dx.doi.org/10.1016/j.tra.2013.01.009.

Deci, E. L., \& Ryan, R. M. (1985). Intrinsic motivation and self-determination in human behavior. New York: Plenum Press.

Diamantopoulos, A., Schlegelmilch, B. B., Sinkovics, R. R., \& Bohlen, G. M. (2003). Can socio-demographics still play a role in profiling green consumers? A review of the evidence and an empirical investigation. Journal of Business research, 56(6), 465-480. http://dx.doi.org/10.1016/S0148-2963(01)00241-7.

Dittmar, H. (1992). The social psychology of material possessions: To have is to be. Hemel Hempstead, UK: Harvester Wheatsheaf.

Donald, I. J., Cooper, S. R., \& Conchie, S. M. (2014). An extended theory of planned behaviour model of the psychological factors affecting commuters' transport mode use. Journal of Environmental Psychology, 40(December), 39-48. http://dx.doi.org/10.1016/j.jenvp.2014.03.003.

Dulal, H. B., \& Akbar, S. (2013). Greenhouse gas emission reduction options for cities: Finding the "Coincidence of Agendas" between local priorities and climate change mitigation objectives. Habitat International, 38(April), 100-105. http://dx.doi.org/10.1016/j.habitatint.2012.05.001.

Dunlap, R. E., \& Van Liere, K. D. (1978). The "new environmental paradigm". The Journal of Environmental Education, 9(4), 10-19. http://dx.doi.org/10.1080/ 00958964.1978.10801875.

Dunlap, R. E., Van Liere, K. D., Mertig, A. G., \& Jones, R. E. (2000). New trends in measuring environmental attitudes: Measuring endorsement of the new ecological paradigm: A revised NEP scale. Journal of Social Issues, 56(3), 425-442. http://dx.doi.org/10.1111/0022-4537.00176.

EPA (2016). Inventory of U.S. Greenhouse Gas Emissions and Sinks: 1990-2014. U.S. Environmental Protection Agency. Retrieved from <https://www3. epa.gov/climatechange/ghgemissions/usinventoryreport.html>.

Eriksson, L., \& Forward, S. E. (2011). Is the intention to travel in a pro-environmental manner and the intention to use the car determined by different factors? Transportation Research Part D: Transport and Environment, 16(5), 372-376. http://dx.doi.org/10.1016/j.trd.2011.02.003.

Eriksson, L., Garvill, J., \& Nordlund, A. M. (2006). Acceptability of travel demand management measures: The importance of problem awareness, personal norm, freedom, and fairness. Journal of Environmental Psychology, 26(1), 15-26. http://dx.doi.org/10.1016/j.jenvp.2006.05.003.

Eriksson, L., Garvill, J., \& Nordlund, A. M. (2008). Interrupting habitual car use: The importance of car habit strength and moral motivation for personal car use reduction. Transportation Research Part F: Traffic Psychology and Behaviour, 11(1), 10-23. http://dx.doi.org/10.1016/j.trf.2007.05.004.

European Commission (2009). Directive 2009/30/EC.

Festinger, L. (1954). A theory of social comparison processes. Human Relations, 7(2), 117-140. http://dx.doi.org/10.1177/001872675400700202. 
Field, A. P. (2005). Is the meta-analysis of correlation coefficients accurate when population correlations vary? Psychological Methods, 10(4), 444-467. http:// dx.doi.org/10.1037/1082-989X.10.4.444.

Fishbein, M., \& Ajzen, I. (1975). Belief, attitude, intention and behavior: An introduction to theory and research. Reading, MA: Addison-Wesley.

Forward, S. E. (2014). Exploring people's willingness to bike using a combination of the theory of planned behavioural and the transtheoretical model. Revue Européenne de Psychologie Appliquée/European Review of Applied Psychology, 64(3), 151-159. http://dx.doi.org/10.1016/j.erap.2014.04.002.

Frey, B. S. (1988). Ipsative and objective limits to human behavior. Journal of Behavioral Economics, 17(4), $229-248$.

Friedrichsmeier, T., Matthies, E., \& Klöckner, C. A. (2013). Explaining stability in travel mode choice: An empirical comparison of two concepts of habit. Transportation Research Part F: Traffic Psychology and Behaviour, 16(January), 1-13. http://dx.doi.org/10.1016/j.trf.2012.08.008.

Fujii, S. (2006). Environmental concern, attitude toward frugality, and ease of behavior as determinants of pro-environmental behavior intentions. Journal of Environmental Psychology, 26(4), 262-268. http://dx.doi.org/10.1016/j.jenvp.2006.09.003.

Gambhir, A., Lawrence, K. C., Tong, D., \& Martinez-Botas, R. (2015). Reducing China's road transport sector $\mathrm{CO}_{2}$ emissions to 2050: Technologies, costs and decomposition analysis. Applied Energy, 157(November), 905-917. http://dx.doi.org/10.1016/j.apenergy.2015.01.018.

Gardner, B. (2009). Modelling motivation and habit in stable travel mode contexts. Transportation Research Part F: Traffic Psychology and Behaviour, 12(1), 68-76. http://dx.doi.org/10.1016/j.trf.2008.08.001.

Gardner, B., \& Abraham, C. (2008). Psychological correlates of car use: A meta-analysis. Transportation Research Part F: Traffic Psychology and Behaviour, 11(4), 300-311. http://dx.doi.org/10.1016/j.trf.2008.01.004.

Gardner, B., \& Abraham, C. (2010). Going green? Modeling the impact of environmental concerns and perceptions of transportation alternatives on decisions to drive. Journal of Applied Social Psychology, 40(4), 831-849. http://dx.doi.org/10.1111/j.1559-1816.2010.00600.x.

Gardner, G. T., \& Stern, P. C. (2008). The short list: The most effective actions US households can take to curb climate change. Environment: Science and Policy for Sustainable Development, 50(5), 12-25. http://dx.doi.org/10.3200/ENVT.50.5.12-25.

Gärling, T., \& Axhausen, K. W. (2003). Introduction: Habitual travel choice. Transportation, 30(1), 1-11. http://dx.doi.org/10.1023/A:1021230223001.

Gärling, T., Fujii, S., \& Boe, O. (2001). Empirical tests of a model of determinants of script-based driving choice. Transportation Research Part F: Traffic Psychology and Behaviour, 4(2), 89-102. http://dx.doi.org/10.1016/S1369-8478(01)00016-X.

Garvill, J., Marell, A., \& Nordlund, A. (2003). Effects of increased awareness on choice of travel mode. Transportation, 30(1), 63-79. http://dx.doi.org/10.1023/ A:1021286608889.

Gatersleben, B. (2011). The car as a material possession: Exploring the link between materialism and car ownership and use. Auto Motives: Understanding Car Use Behaviors, 137-148. http://dx.doi.org/10.1108/9780857242341-007.

Geurs, K. T., \& Van Wee, B. (2004). Accessibility evaluation of land-use and transport strategies: Review and research directions. Journal of Transport Geography, 12(2), 127-140. http://dx.doi.org/10.1016/j.jtrangeo.2003.10.005.

Hao, H., Geng, Y., Li, W., \& Guo, B. (2015). Energy consumption and GHG emissions from China's freight transport sector: Scenarios through 2050. Energy Policy, 85(October), 94-101. http://dx.doi.org/10.1016/j.enpol.2015.05.016.

Hao, H., Liu, Z., Zhao, F., Li, W., \& Hang, W. (2015). Scenario analysis of energy consumption and greenhouse gas emissions from China's passenger vehicles. Energy, 91(November), 151-159. http://dx.doi.org/10.1016/j.energy.2015.08.054.

Harland, P., Staats, H., \& Wilke, H. A. (1999). Explaining proenvironmental intention and behavior by personal norms and the theory of planned behavior. Journal of Applied Social Psychology, 29(12), 2505-2528. http://dx.doi.org/10.1111/j.1559-1816.1999.tb00123.x.

Haustein, S., \& Hunecke, M. (2007). Reduced use of environmentally friendly modes of transportation caused by perceived mobility necessities: An extension of the theory of planned behavior. Journal of Applied Social Psychology, 37(8), 1856-1883. http://dx.doi.org/10.1111/j.1559-1816.2007.00241. $\mathrm{x}$.

Haustein, S., Klöckner, C. A., \& Blöbaum, A. (2009). Car use of young adults: The role of travel socialization. Transportation Research Part F: Traffic Psychology and Behaviour, 12(2), 168-178. http://dx.doi.org/10.1016/j.trf.2008.10.003.

Heath, Y., \& Gifford, R. (2002). Extending the theory of planned behavior: Predicting the use of public transportation. Journal of Applied Social Psychology, 32 (10), 2154-2189. http://dx.doi.org/10.1111/j.1559-1816.2002.tb02068.x.

Hedges, L. V., \& Olkin, I. (1985). Statistical methods for meta-analysis. Orlando, FL: Academic Press.

Hedges, L. V., \& Vevea, J. L. (1998). Fixed-and random-effects models in meta-analysis. Psychological Methods, 3(4), 486-504. http://dx.doi.org/10.1037/ 1082-989X.3.4.486.

Higgins, J. P. T., Thompson, S. G., Deeks, J. J., \& Altman, D. G. (2003). Measuring inconsistency in meta-analyses. British Medical Journal, 327, 557-560. http:// dx.doi.org/10.1136/bmj.327.7414.557.

Hsiao, C. H., \& Yang, C. (2010). Predicting the travel intention to take High Speed Rail among college students. Transportation Research Part F: Traffic Psychology and Behaviour, 13(4), 277-287. http://dx.doi.org/10.1016/j.trf.2010.04.011.

Hunecke, M., Haustein, S., Böhler, S., \& Grischkat, S. (2010). Attitude-based target groups to reduce the ecological impact of daily nobility behavior. Environment and Behavior, 42(1), 3-43. http://dx.doi.org/10.1177/0013916508319587.

IPCC (2014). Climate Change 2014: Synthesis Report. Contribution of Working Groups I, II and III to the Fifth Assessment Report of the Intergovernmental Panel on Climate Change. Retrieved from <http://www.ipcc.ch/pdf/assessment-report/ar5/syr/AR5_SYR_FINAL_All_Topics.pdf>.

Joireman, J. A., Van Lange, P. A., Kuhlman, D. M., Van Vugt, M., \& Shelley, G. P. (1997). An interdependence analysis of commuting decisions. European Journal of Social Psychology, 27(4), 441-463.

Kaiser, F. G., Ranney, M., Hartig, T., \& Bowler, P. A. (1999). Ecological behavior, environmental attitude, and feelings of responsibility for the environment. European Psychologist, 4(2), 59-74. http://dx.doi.org/10.1027//1016-9040.4.2.59.

Kennedy, E. H., Beckley, T. M., McFarlane, B. L., \& Nadeau, S. (2009). Why we don't "walk the talk": Understanding the environmental values/behaviour gap in Canada. Human Ecology Review, 16(2), 151-160.

Kim, S., \& Ulfarsson, G. (2008). Curbing automobile use for sustainable transportation: Analysis of mode choice on short home-based trips. Transportation, 35 (6), 723-737. http://dx.doi.org/10.1007/s11116-008-9177-5.

Klöckner, C. A. (2004). How single events change travel mode choice-a life span perspective. In 3rd International Conference on Traffic E̊ Transport Psychology, Nottingham, UK.

Klöckner, C. A., \& Matthies, E. (2009). Structural modeling of car use on the way to the university in different settings: Interplay of norms, habits, situational restraints, and perceived behavioral control. Journal of Applied Social Psychology, 39(8), 1807-1834. http://dx.doi.org/10.1111/j.1559-1816.2009.00505.x.

Kohlberg, L. (1984). Essays on moral development. The psychology of moral development (Vol. 2) New York: Harper \& Row Publishers Inc.

Kollmuss, A., \& Agyeman, J. (2002). Mind the gap: Why do people act environmentally and what are the barriers to pro-environmental behavior? Environmental Education Research, 8(3), 239-260. http://dx.doi.org/10.1080/13504620220145401.

Lai, I. K., Liu, Y., Sun, X., Zhang, H., \& Xu, W. (2015). Factors influencing the behavioural intention towards full electric vehicles: An empirical study in Macau. Sustainability, 7(9), 12564-12585. http://dx.doi.org/10.3390/su70912564.

Lane, B., \& Potter, S. (2007). The adoption of cleaner vehicles in the UK: Exploring the consumer attitude-action gap. Journal of Cleaner Production, 15(11), 1085-1092. http://dx.doi.org/10.1016/j.jclepro.2006.05.026.

Lanzendorf, M. (2003). Mobility biographies. A new perspective for understanding travel behaviour. In: Paper Presented at the 10th International Conference on Travel Behaviour Research (IATBR), Lucerne, 10-15 August, 2003.

Lanzendorf, M. (2010). Key events and their effect on mobility biographies: The case of childbirth. International Journal of Sustainable Transportation, 4(5), 272-292. http://dx.doi.org/10.1080/15568310903145188.

Lanzini, P., \& Thøgersen, J. (2014). Behavioural spillover in the environmental domain: An intervention study. Journal of Environmental Psychology, 40 (December), 381-390. http://dx.doi.org/10.1016/j.jenvp.2014.09.006. 
Lee, H. S., \& Shepley, M. M. (2012). Perceived neighborhood environments and leisure-time walking among Korean adults: An application of the theory of planned behavior. HERD Journal, 5(2), 99-110. http://dx.doi.org/10.1177/193758671200500208.

Limtanakool, N., Dijst, M., \& Schwanen, T. (2006). The influence of socioeconomic characteristics, land use and travel time considerations on mode choice for medium-and longer-distance trips. Journal of Transport Geography, 14(5), 327-341. http://dx.doi.org/10.1016/j.jtrangeo.2005.06.004.

Litman, T. (2005). Land use impacts on transport: How land use factors affect travel behavior. Victoria: Victoria Transport Institute. Retrieved from <http:// www.vtpi.org/landtravel.pdf>.

Lo, S. H., van Breukelen, G. J., Peters, G. J. Y., \& Kok, G. (2016). Commuting travel mode choice among office workers: Comparing an Extended Theory of Planned Behavior model between regions and organizational sectors. Travel Behaviour and Society, 4(May), 1-10. http://dx.doi.org/10.1016/j. tbs.2015.11.002.

Lois, D., Moriano, J. A., \& Rondinella, G. (2015). Cycle commuting intention: A model based on theory of planned behaviour and social identity. Transportation Research Part F: Traffic Psychology and Behaviour, 32(July), 101-113. http://dx.doi.org/10.1016/j.trf.2015.05.003.

Loukopoulos, P., \& Gärling, T. (2005). Are car users too lazy to walk?: The relationship of distance thresholds for driving to the perceived effort of walking. Transportation Research Record: Journal of the Transportation Research Board, 1926, 206-211. http://dx.doi.org/10.3141/1926-24.

Mann, E., \& Abraham, C. (2012). Identifying beliefs and cognitions underpinning commuters' travel mode choices. Journal of Applied Social Psychology, 42(11), 2730-2757. http://dx.doi.org/10.1111/j.1559-1816.2012.00959.x.

Manstead, A. S., \& Parker, D. (1995). Evaluating and extending the theory of planned behaviour. European Review of Social Psychology, 6(1), 69-95. http://dx. doi.org/10.1080/14792779443000012.

Masters, J. C., \& Smith, W. P. (1987). Social comparison, social justice, and relative deprivation: Theoretical, empirical, and policy perspectives. Hillsdale, NJ: Lawrence Erlbaum Associates, Inc..

Mokhtarian, P. L., \& Cao, X. (2008). Examining the impacts of residential self-selection on travel behavior: A focus on methodologies. Transportation Research Part B: Methodological, 42(3), 204-228. http://dx.doi.org/10.1016/j.trb.2007.07.006.

Möser, G., \& Bamberg, S. (2008). The effectiveness of soft transport policy measures: A critical assessment and meta-analysis of empirical evidence. Journal of Environmental Psychology, 28(1), 10-26. http://dx.doi.org/10.1016/j.jenvp.2007.09.001.

Murtagh, S., Rowe, D. A., Elliott, M. A., McMinn, D., \& Nelson, N. M. (2012). Predicting active school travel: The role of planned behavior and habit strength. International Journal of Behavioral Nutrition and Physical Activity, 9(1), 65-74. http://dx.doi.org/10.1186/1479-5868-9-65.

Nilsson, M., \& Küller, R. (2000). Travel behaviour and environmental concern. Transportation Research Part D: Transport and Environment, 5(3), 211-234. http://dx.doi.org/10.1016/S1361-9209(99)00034-6.

Noblet, C. L., Thøgersen, J., \& Teisl, M. F. (2014). Who attempts to drive less in New England? Transportation Research Part F: Traffic Psychology and Behaviour, 23(March), 69-80. http://dx.doi.org/10.1016/j.trf.2013.12.016.

Nordfjærn, T., Şimşekoğlu, Ö., \& Rundmo, T. (2014). The role of deliberate planning, car habit and resistance to change in public transportation mode use. Transportation Research Part F: Traffic Psychology and Behaviour, 27(November), 90-98. http://dx.doi.org/10.1016/j.trf.2014.09.010.

Nordlund, A. M., \& Garvill, J. (2002). Value structures behind proenvironmental behavior. Environment and Behavior, 34(6), 740-756. http://dx.doi.org/ $10.1177 / 001391602237244$.

Nordlund, A. M., \& Garvill, J. (2003). Effects of values, problem awareness, and personal norm on willingness to reduce personal car use. Journal of Environmental Psychology, 23(4), 339-347. http://dx.doi.org/10.1016/S0272-4944(03)00037-9.

Nordlund, A., \& Westin, K. (2013). Influence of values, beliefs, and age on intention to travel by a new railway line under construction in northern Sweden. Transportation Research Part A: Policy and Practice, 48(February), 86-95. http://dx.doi.org/10.1016/j.tra.2012.10.008.

O'Fallon, C., Sullivan, C., \& Hensher, D. A. (2004). Constraints affecting mode choices by morning car commuters. Transport Policy, 11(1), 17-29. http://dx.doi. org/10.1016/S0967-070X(03)00015-5.

Onwezen, M. C., Antonides, G., \& Bartels, J. (2013). The Norm Activation Model: An exploration of the functions of anticipated pride and guilt in proenvironmental behaviour. Journal of Economic Psychology, 39, 141-153. http://dx.doi.org/10.1016/j.joep.2013.07.005.

Oskamp, S. (2000). A sustainable future for humanity? How can psychology help? American Psychologist, 55(5), 496-508. http://dx.doi.org/10.1037/0003066X.55.5.496.

Ouellette, J. A., \& Wood, W. (1998). Habit and intention in everyday life: The multiple processes by which past behavior predicts future behavior. Psychological Bulletin, 124(1), 54-74. http://dx.doi.org/10.1037/0033-2909.124.1.54.

Parker, D., Manstead, A. S., \& Stradling, S. G. (1995). Extending the theory of planned behaviour: The role of personal norm. British Journal of Social Psychology, 34(2), 127-138. http://dx.doi.org/10.1111/j.2044-8309.1995.tb01053.x.

Passafaro, P., Rimano, A., Piccini, M. P., Metastasio, R., Gambardella, V., Gullace, G., \& Lettieri, C. (2014). The bicycle and the city: Desires and emotions versus attitudes, habits and norms. Journal of Environmental Psychology, 38(June), 76-83. http://dx.doi.org/10.1016/j.jenvp.2013.12.011.

Perugini, M., \& Bagozzi, R. P. (2001). The role of desires and anticipated emotions in goal-directed behaviours: Broadening and deepening the theory of planned behaviour. British Journal of Social Psychology, 40(1), 79-98. http://dx.doi.org/10.1348/014466601164704.

Peters, A., von Klot, S., Heier, M., Trentinaglia, I., Hörmann, A., Wichmann, H. E., \& Löwel, H. (2004). Exposure to traffic and the onset of myocardial infarction. New England Journal of Medicine, 351(17), 1721-1730. http://dx.doi.org/10.1056/NEJMoa040203.

Polk, M. (2003). Are women potentially more accommodating than men to a sustainable transportation system in Sweden? Transportation Research Part D: Transport and Environment, 8(2), 75-95. http://dx.doi.org/10.1016/S1361-9209(02)00034-2.

Prillwitz, J., Harms, S., \& Lanzendorf, M. (2006). Impact of life-course events on car ownership. Transportation Research Record: Journal of the Transportation Research Board, 1985, 71-77. http://dx.doi.org/10.3141/1985-08.

Pucher, J., \& Renne, J. L. (2003). Socioeconomics of urban travel: Evidence from the 2001 NHTS. Transportation Quarterly, 57(3), 49-77.

Ronis, D. L., Yates, J. F., \& Kirscht, J. P. (1989). Attitudes, decisions, and habits as determinants of repeated behavior. In A. R. Pratkanis, S. J. Breckler, \& A. G. Greenwald (Eds.), Attitude structure and function (pp. 213-239). Hillsdale, NJ: Lawrence Erlbaum.

Rosenthal, R. (1984). Meta-analytic procedures for social research. London: Sage.

Scheiner, J. (2007). Mobility biographies: Elements of a biographical theory of travel demand. Erdkunde, 61, 161-173. Retrieved from: <http://www.jstor. org/stable/25647982>.

Scheiner, J., \& Holz-Rau, C. (2013). Changes in travel mode use after residential relocation: A contribution to mobility biographies. Transportation, 40(2), 431-458. http://dx.doi.org/10.1007/s11116-012-9417-6.

Schlenker, B. R. (1980). Impression management: The self-concept, social identity, and interpersonal relations. Monterey, CA: Brooks/Cole Publishing Company.

Schoenduwe, R., Mueller, M. G., Peters, A., \& Lanzendorf, M. (2015). Analysing mobility biographies with the life course calendar: A retrospective survey methodology for longitudinal data collection. Journal of Transport Geography, 42, 98-109. http://dx.doi.org/10.1016/j.jtrangeo.2014.12.001.

Schuitema, G., Steg, L., \& Forward, S. (2010). Explaining differences in acceptability before and acceptance after the implementation of a congestion charge in Stockholm. Transportation Research Part A: Policy and Practice, 44(2), 99-109. http://dx.doi.org/10.1016/j.tra.2009.11.005.

Schwartz, S. H. (1977). Normative influences on altruism. Advances in Experimental Social Psychology, 10, 221-279. http://dx.doi.org/10.1016/S0065-2601 (08)60358-5.

Schwartz, S. H. (1992). Universals in the content and structure of values: Theoretical advances and empirical tests in 20 countries. Advances in Experimental Social Psychology, 25, 1-65. http://dx.doi.org/10.1016/S0065-2601(08)60281-6.

Schwartz, S. H. (1994). Are there universal aspects in the structure and contents of human values? Journal of Social Issues, 50(4), 19-45.

Schwartz, S. H., \& Howard, J. A. (1981). A normative decision-making model of altruism. In J. P. Rushton (Ed.), Altruism and helping behaviour: Social, personality and developmental perspectives (pp. 189-211). Hillsdale, NJ: Erlbaum.

Sheeran, P. (2002). Intention-Behavior relations: A conceptual and empirical review. European Review of Social Psychology, 12(1), 1-36. http://dx.doi.org/ $10.1080 / 14792772143000003$. 
Sheppard, B. H., Hartwick, J., \& Warshaw, P. R. (1988). The theory of reasoned action: A meta-analysis of past research with recommendations for modifications and future research. Journal of Consumer Research, 15(3), 325-343. http://dx.doi.org/10.1086/209170.

Staats, H., Harland, P., \& Wilke, H. A. (2004). Effecting durable change a team approach to improve environmental behavior in the household. Environment and Behavior, 36(3), 341-367. http://dx.doi.org/10.1177/0013916503260163.

Steg, L. (2005). Car use: Lust and must. Instrumental, symbolic and affective motives for car use. Transportation Research Part A: Policy and Practice, 39(2-3), 147-162. http://dx.doi.org/10.1016/j.tra.2004.07.001.

Steg, L. (2004). Car use: Lust and must. In T. Rothengatter \& R. D. Huguenin (Eds.), Traffic and transport psychology (pp. 443-452). Amsterdam: Elseveir.

Steg, L., \& Sievers, I. (2000). Cultural theory and individual perceptions of environmental risks. Environment and Behavior, 32(2), 250-269. http://dx.doi.org/ $10.1177 / 00139160021972513$.

Stern, P. C. (2000). New environmental theories: Toward a coherent theory of environmentally significant behavior. Journal of Social Issues, 56(3), 407-424. http://dx.doi.org/10.1111/0022-4537.00175.

Stern, P. C. (2011). Contributions of psychology to limiting climate change. American Psychologist, 64(4), 303-314. http://dx.doi.org/10.1037/a0023235.

Stern, P. C., Dietz, T., Abel, T. D., Guagnano, G. A., \& Kalof, L. (1999). A value-belief-norm theory of support for social movements: The case of environmentalism. Human Ecology Review, 6(2), 81-97.

Sutton, S. (1998). Predicting and explaining intentions and behavior: How well are we doing? Journal of Applied Social Psychology, 28(15), 1317-1338. http:// dx.doi.org/10.1111/j.1559-1816.1998.tb01679.x.

Tanner, C. (1999). Constraints on environmental behavior. Journal of Environmental Psychology, 19(2), 145-157. http://dx.doi.org/10.1006/jevp.1999.0121.

Thøgersen, J. (2006). Norms for environmentally responsible behaviour: An extended taxonomy. Journal of Environmental Psychology, 26(4), 247-261. http:// dx.doi.org/10.1016/j.jenvp.2006.09.004.

Triandis, H. C. (1977). Interpersonal behavior. Monterey, CA: Brooks/Cole.

Triandis, H. C. (1980). Values, attitudes, and interpersonal behavior. In H. E. Howe \& M. M. Page (Eds.), Nebraska symposium on motivation 1979 (pp. 195-259). Lincoln: University of Nebraska Press.

Van Acker, V., Van Wee, B., \& Witlox, F. (2010). When transport geography meets social psychology: Toward a conceptual model of travel behaviour. Transport Reviews, 30(2), 219-240. http://dx.doi.org/10.1080/01441640902943453.

Van der Waerden, P. J. H. J., Borgers, A. W. J., \& Timmermans, H. J. P. (2003). Key events and critical incidents influencing transport mode choice switching behavior: an exploratory study. In Proceedings 82nd annual meeting of the transportation research board (pp. 12-16), Washington, DC.

Van Vugt, M., Meertens, R. M., \& Lange, P. A. (1995). Car versus public transportation? The role of social value orientations in a real-life social dilemma. Journal of Applied Social Psychology, 25(3), 258-278. http://dx.doi.org/10.1111/j.1559-1816.1995.tb01594.x.

Verplanken, B., \& Aarts, H. (1999). Habit, attitude, and planned behaviour: Is habit an empty construct or an interesting case of goal-directed automaticity? European Review of Social Psychology, 10(1), 101-134. http://dx.doi.org/10.1080/14792779943000035.

Verplanken, B., Aarts, H., van Knippenberg, A., \& Moonen, A. (1998). Habit versus planned behaviour: A field experiment. The British Journal of Social Psychology, 37, 111-128. http://dx.doi.org/10.1111/j.2044-8309.1998.tb01160.x.

Verplanken, B., Aarts, H., van Knippenberg, A., \& van Knippenberg, C. (1994). Attitude versus general habit: Antecedents of travel mode Choice. Journal of Applied Social Psychology, 24(4), 285-300. http://dx.doi.org/10.1111/j.1559-1816.1994.tb00583.x.

Verplanken, B., Walker, I., Davis, A., \& Jurasek, M. (2008). Context change and travel mode choice: Combining the habit discontinuity and self-activation hypotheses. Journal of Environmental Psychology, 28(2), 121-127. http://dx.doi.org/10.1016/j.jenvp.2007.10.005.

Verplanken, B., \& Wood, W. (2006). Interventions to break and create consumer habits. Journal of Public Policy E Marketing, 25(1), 90-103. http://dx.doi.org/ 10.1509/jppm.25.1.90.

Winship, C., \& Morgan, S. L. (1999). The estimation of causal effects from observational data. Annual Review of Sociology, 25, 659-706. Retrieved from: <http://www.jstor.org/stable/223520>.

Yang-Wallentin, F., Schmidt, P., Davidov, E., \& Bamberg, S. (2004). Is there any interaction effect between intention and perceived behavioral control? Methods of Psychological Research Online, 8(2), 127-157. 